Ilari Hänninen, Mikko Pitkonen, Keijo I. Nikoskinen, and Jukka Sarvas, Method of moments analysis of the backscattering properties of a corrugated trihedral corner reflector, IEEE Transactions on Antennas and Propagation, Vol. 54, no. 4, pp. 1167-1173, 2006.

(C) 2006 IEEE

Reprinted with permission.

This material is posted here with permission of the IEEE. Such permission of the IEEE does not in any way imply IEEE endorsement of any of Helsinki University of Technology's products or services. Internal or personal use of this material is permitted. However, permission to reprint/republish this material for advertising or promotional purposes or for creating new collective works for resale or redistribution must be obtained from the IEEE by writing to pubs-permissions@ieee.org.

By choosing to view this document, you agree to all provisions of the copyright laws protecting it. 


\title{
Method of Moments Analysis of the Backscattering Properties of a Corrugated Trihedral Corner Reflector
}

\author{
Ilari Hänninen, Mikko Pitkonen, Keijo I. Nikoskinen, Senior Member, IEEE, and Jukka Sarvas, Member, IEEE
}

\begin{abstract}
A method of moments (MoM) formulation is developed to analyze the backscattering properties of an anisotropic trihedral corner reflector, which is obtained by corrugating one or several of its interior faces. The proposed formulation treats the corrugated surface as ideally tuned to the incident wave frequency. The numerical analysis of the studied structures has been done using closed-form formulas and accurate numerical integration. The focus of the study reported in this paper has been the polarization responses of ideally tuned corrugated reflectors, which have interesting properties, particularly regarding elliptically or circularly polarized waves. We numerically verify that an appropriately corrugated reflector returns elliptically and circularly polarized waves with the same handedness as the incident wave. For a linearly polarized incident wave, the corner reflector is able to rotate them by $90^{\circ}$. Also the effect of the direction of the corrugation to the backscattering properties is studied.
\end{abstract}

Index Terms-Corner reflector, electromagnetic scattering, method of moments (MoM), radar cross-section (RCS), soft and hard surface (SHS).

\section{INTRODUCTION}

$\mathbf{T}$ RIHEDRAL corner reflectors are widely used as location markers and calibration targets in radar technology and remote sensing. They have a high backscattering radar cross-section over a wide angular range, they do not require any power to operate, they are mechanically easy to construct, and they can also be operated in difficult conditions. Conventional trihedral corner reflectors, i.e., reflectors made from a perfect electric conductor (PEC) material return linearly polarized incident electromagnetic waves with the same polarization but they reverse the handedness of elliptically and circularly polarized incident waves. Depolarizing trihedral corner reflectors, however, retain the handedness of elliptically and circularly polarized incident waves, which is an important factor when one wishes to use polarimetric radars. Depolarizing corner reflectors are also able to rotate linearly polarized incident waves by $90^{\circ}$. These properties also make the reflected wave easy to distinguish from the background noise due the environment. In this paper, we consider a trihedral corner reflector (Fig. 1) where the depolarization is obtained by choosing one or several of the interior faces to be ideally tuned corrugated surfaces.

Ideally tuned corrugated surfaces are a well-known realization of soft and hard surfaces (SHSs) [1]-[3], although soft and hard surfaces can also be realized by other means [4], [5]. The corrugation is called ideally tuned if its geometric dimensions

Manuscript received June 21, 2005; revised December 2, 2005.

The authors are with the Electromagnetics Laboratory, Helsinki University of Technology, FI-02015, Espoo, Finland (e-mail: ilari.hanninen@tkk.fi).

Digital Object Identifier 10.1109/TAP.2006.872676

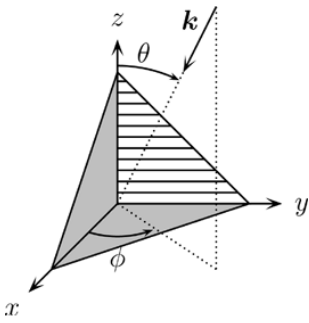

Fig. 1. Geometry of the trihedral corner reflector with a corrugated interior face.

are chosen in such a way that the corrugated surface presents an ideal SHS for the incident electromagnetic wave for a selected wavelength. The SHSs have the property that the reflected fields from them retain the incident polarization. Then the ray theory predicts that the reflection from the trihedral corner reflector either retains or reverses the polarization according to how many of the inside faces of the corner reflector are made of SHS plates and how many are made of PEC plates. The use of SHSs in trihedral corner reflectors has been previously discussed, but a rigorous numerical analysis of such structures has been lacking [6]-[8]. In this paper, a method of moments (MoM) formulation is derived for an ideally tuned corrugated surface. The presented formulation can also be used for mixed structures, which contain both PEC and SHS domains. The results of the MoM are verified using a physical optics (PO) method.

\section{IDEALLY TUNED CORRUGATION}

SHS has been introduced as a convenient idealized model for the ideally tuned corrugated surface. They have the unique property that they have the same boundary conditions for both electric and magnetic fields. Let us mark by $\hat{\boldsymbol{u}}$ the tangential unit vector to the direction of the corrugation (i.e., the conducting direction) and by $\hat{\boldsymbol{v}}$ the tangential unit vector perpendicular to the direction of the corrugation (i.e., the nonconducting direction). Then the boundary conditions for the electric and magnetic fields on the SHS can be written [3]

$$
\hat{\boldsymbol{u}} \cdot \boldsymbol{E}=0, \quad \hat{\boldsymbol{u}} \cdot \boldsymbol{H}=0
$$

and so the SHS can be regarded as a combined PEC and perfect magnetic conductor (PMC) surface to the direction of the corrugation. An equivalent way to achieve an SHS is to require that the surface impedance $Z_{u}$ seen by the electric field to the direction of the corrugation approaches zero and that the surface impedance $Z_{v}$ perpendicular to the direction of the corrugation 


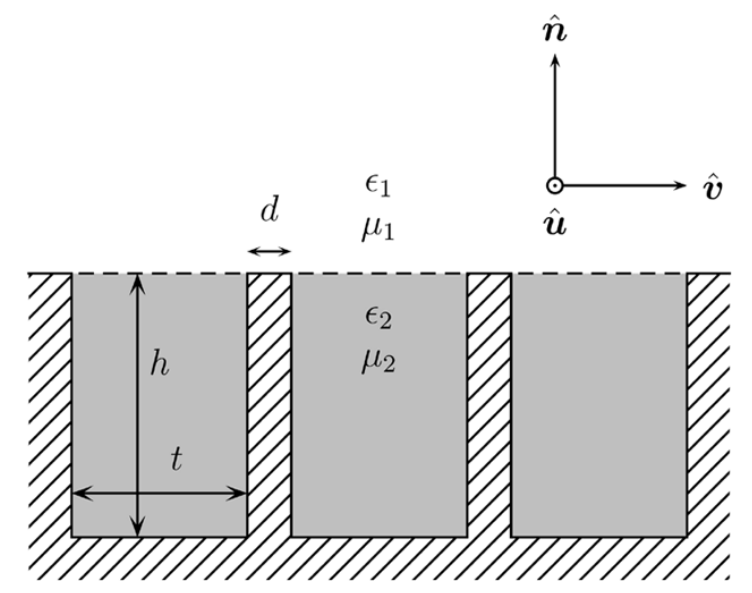

Fig. 2. The physical properties of the corrugation.

approaches infinity [1]. We define the surface impedances $Z_{u}$ and $Z_{v}$ by the following equations:

$$
Z_{u}=\frac{E_{u}}{H_{v}}, \quad Z_{v}=\frac{E_{v}}{H_{u}}
$$

where $E_{\{u, v\}}$ and $H_{\{u, v\}}$ are the components of the electric and magnetic field to the direction parallel or perpendicular to the corrugation, respectively.

In practice, an ideally tuned corrugated surface, which reasonably well simulates an SHS, can be achieved as follows; see, e.g., [9]. Let the geometrical properties of the corrugation be as indicated in Fig. 2, with the height of the corrugation denoted by $h$, the width by $t$, and the thickness of the walls between the grooves by $d$. We assume that the width of the corrugation is much smaller than the incident wavelength $\lambda_{1}$ and also that the distance separating the grooves is much smaller than the width of the corrugation, i.e.,

$$
d \ll t+d \ll \lambda_{1} / 2 \sqrt{\epsilon_{2} / \epsilon_{1}}
$$

where $\epsilon_{2}$ is the permittivity inside the grooves and $\epsilon_{1}$ is the permittivity outside the scatterer. Then, if the incident wave is a TM-wave (i.e., the incident electric field vector is in the plane formed by the surface normal $\hat{\boldsymbol{n}}$ and the conducting direction $\hat{\boldsymbol{u}})$, the dominating waveform created inside the corrugation is an evanescent $\mathrm{TE}_{1}$-waveform. The surface impedance seen by the incident electric field component $E_{u}$ can then be written

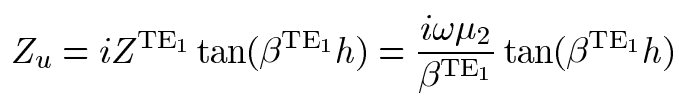

where $Z^{\mathrm{TE}_{1}}=\omega \mu_{2} / \beta^{\mathrm{TE}_{1}}$ is the wave impedance for the $\mathrm{TE}_{1}$-waveform, $\beta^{\mathrm{TE}_{1}}$ is the propagation factor, and $k_{2}=\left|k_{2}\right|$ and $\mu_{2}$ are the wave number and permeability inside the corrugation. Because $k_{2} t \ll \pi$, we can approximate the propagation factor $\beta^{\mathrm{TE}_{1}}$ by

$$
\beta^{\mathrm{TE}_{1}}=\frac{1}{t} \sqrt{\left(k_{2} t\right)^{2}-\pi^{2}} \approx i \frac{\pi}{t}
$$

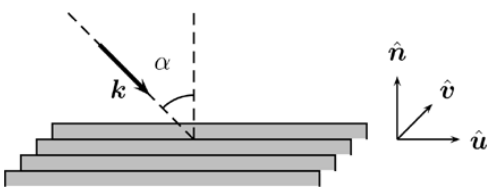

Fig. 3. If the incident TE-wave arrives to the surface travelling to the direction of the corrugation, i.e., in the un-plane, the surface impedance seen by the incident electric field is highly dependent of the incident angle $\alpha$. This dependency can be compensated for by filling the corrugation with a dielectric material such that $\epsilon_{2} \gg \epsilon_{1}$.

and $\tan \left(\beta^{\mathrm{TE}} h\right)$ by

$$
\tan \left(\beta^{\mathrm{TE}_{1}} h\right)=\tan (i \pi h / t)=\frac{2 i}{2} \frac{e^{-\pi h / t}-e^{\pi h / t}}{e^{-\pi h / t}+e^{\pi h / t}} \approx i .
$$

Then the surface impedance component parallel to the corrugation becomes

$$
Z_{u} \approx i \frac{i \omega \mu_{2} t}{i \pi}=i \frac{k_{2} t \eta_{2}}{\pi}, \quad \eta_{2}=\sqrt{\frac{\mu_{2}}{\epsilon_{2}}}
$$

which approaches zero as $k_{2} t$ approaches zero, i.e., when the width $t$ of the grooves becomes very small compared to the wavelength.

Strictly speaking, for (4) for the surface impedance component $Z_{u}$ to be correct, it is assumed that the incident wave arrives to the surface from the normal direction. In reality the surface impedance $Z_{u}$ depends on the incident angle of the illuminating wave. However, for incident TM-waves, the dependency on the incident angle is insignificant if the width of the corrugation is very small compared to the wavelength, as we have assumed.

If the incident wave is a TE-wave (i.e., the incident electric field vector is in the plane formed by the surface normal $\hat{\boldsymbol{n}}$ and the nonconducting vector $\hat{\boldsymbol{v}}$ ), the electric field component $E_{v}$ sees a surface impedance

$$
Z_{v}=i \eta_{2} \tan \left(k_{2 n} h\right)
$$

where $k_{2 n}=\left|\hat{\boldsymbol{n}} \cdot \boldsymbol{k}_{2}\right|$. This surface impedance component is dependent on the incident angle $\alpha$, measured from the $n$ axis toward the $u$ axis (see Fig. 3). When the incident wave arrives to the surface at an angle $\alpha>0$, the path travelled by the incident wave inside the corrugation diverges from the normal direction, which changes the surface impedance $Z_{v}$ seen at the mouth of the grooves. However, it is possible to greatly diminish the directional dependence by filling the grooves by a dielectric material with $\epsilon_{2} / \epsilon_{1} \gg 1$, since then the path of the wave inside the corrugation follows more closely the normal direction as the incident wave is deflected toward the normal. When the height of the corrugation $h$ is chosen as [2]

$$
h=\frac{\lambda_{1}}{4 \sqrt{\left(\epsilon_{2} / \epsilon_{1}\right)-\sin ^{2} \alpha}}
$$

we see that even for grazing incidence, this incident angle dependency can be made quite small. With the height of the corrugation chosen properly, $\tan \left(k_{2 n} h\right)=\infty$, and the surface impedance component perpendicular to the corrugation $Z_{v}$ is infinite. 
If the surface impedances seen by the electric field at the mouth of the grooves satisfy the previous conditions, the top of the corrugation can be replaced by a virtual SHS with equivalent boundary conditions. However, finding the actual physical dimensions of the corrugation required for these conditions to be valid is beyond the scope of this paper. For the rest of this paper, we assume that these conditions hold. Since the SHS requires similar boundary conditions for both the electric field and the magnetic field, there exist on the SHS both equivalent electric and magnetic surface currents. The SHS only allows currents to flow to the direction of the corrugation. This can easily be seen from the boundary conditions (1) as follows. The equivalent electric surface current on the SHS can be written

$$
\begin{aligned}
\boldsymbol{J} & =\hat{\boldsymbol{n}} \times \boldsymbol{H} \\
& =\hat{\boldsymbol{n}} \times\left(H_{n} \hat{\boldsymbol{n}}+H_{v} \hat{\boldsymbol{v}}\right)=H_{v} \hat{\boldsymbol{n}} \times \hat{\boldsymbol{v}} \\
& =-H_{v} \hat{\boldsymbol{u}}
\end{aligned}
$$

and similarly the equivalent magnetic surface current is a function of the tangential electric field

$$
\begin{aligned}
\boldsymbol{M} & =-\hat{\boldsymbol{n}} \times \boldsymbol{E} \\
& =-\hat{\boldsymbol{n}} \times\left(E_{n} \hat{\boldsymbol{n}}+E_{v} \hat{\boldsymbol{v}}\right)=-E_{v} \hat{\boldsymbol{n}} \times \hat{\boldsymbol{v}} \\
& =E_{v} \hat{\boldsymbol{u}} .
\end{aligned}
$$

Thus the equivalent electric and magnetic surface currents can only have $\hat{\boldsymbol{u}}$-components.

\section{METHOD OF MOMENTS FoRMULATION FOR THE IDEALLY TUNED CORRUGATED SURFACE}

The boundary conditions for soft and hard surfaces are nearly similar in expression to the general PEC or PMC surfaces. The PEC, or the PMC, boundary condition states that the tangential electric or magnetic field components are zero on the surface, whereas the SHS boundary condition requires that the tangential field component parallel to the corrugation of both the electric and magnetic field is zero on the surface. Let us denote by $E^{\text {inc }}, H^{\text {inc }}$ the incident electric and magnetic fields and by $\boldsymbol{E}^{\mathrm{s}}, \boldsymbol{H}^{\mathrm{s}}$ the scattered fields, so that the total fields are the sum of the incident and scattered fields. Then the SHS boundary condition requires that for $\boldsymbol{r}$ on the SHS domain

$$
\begin{aligned}
\hat{\boldsymbol{u}}(\boldsymbol{r}) \cdot\left(\boldsymbol{E}^{\mathrm{inc}}(\boldsymbol{r})+\boldsymbol{E}^{\mathrm{s}}(\boldsymbol{r})\right) & =0 \\
\hat{\boldsymbol{u}}(\boldsymbol{r}) \cdot\left(\boldsymbol{H}^{\mathrm{inc}}(\boldsymbol{r})+\boldsymbol{H}^{\mathrm{s}}(\boldsymbol{r})\right) & =0
\end{aligned}
$$

where $\hat{u}(\boldsymbol{r})$ is the direction of the corrugation, i.e., the conducting direction. By using the equivalent surface principle (also called the Stratton-Chu formulas) and the boundary conditions (12) and (13), we can in the usual way derive the electric and magnetic field equations for a closed ideally corrugated surface as

$$
\begin{aligned}
\hat{\boldsymbol{u}}(\boldsymbol{r}) \cdot \boldsymbol{E}^{\mathrm{inc}}(\boldsymbol{r})= & \frac{1}{i \omega \epsilon_{1}} \hat{\boldsymbol{u}}(\boldsymbol{r}) \cdot(\tilde{D} \boldsymbol{J})(\boldsymbol{r}) \\
& +\hat{\boldsymbol{u}}(\boldsymbol{r}) \cdot(\tilde{K} \boldsymbol{M})(\boldsymbol{r})-\frac{1}{2} \hat{\boldsymbol{u}}(\boldsymbol{r}) \cdot \hat{\boldsymbol{n}}(\boldsymbol{r}) \times \boldsymbol{M}(\boldsymbol{r})
\end{aligned}
$$

$$
\begin{aligned}
\hat{\boldsymbol{u}}(\boldsymbol{r}) \cdot \boldsymbol{H}^{\mathrm{inc}}(\boldsymbol{r})= & \frac{1}{i \omega \mu_{1}} \hat{\boldsymbol{u}}(\boldsymbol{r}) \cdot(\tilde{D} \boldsymbol{M})(\boldsymbol{r}) \\
& -\hat{\boldsymbol{u}}(\boldsymbol{r}) \cdot(\tilde{K} \boldsymbol{J})(\boldsymbol{r})+\frac{1}{2} \hat{\boldsymbol{u}}(\boldsymbol{r}) \cdot \hat{\boldsymbol{n}}(\boldsymbol{r}) \times \boldsymbol{J}(\boldsymbol{r})
\end{aligned}
$$

where the integral operators $(\tilde{K} \boldsymbol{F})(\boldsymbol{r})$ and $(\tilde{D} \boldsymbol{F})(\boldsymbol{r})$ on a surface $S$ are defined as

$$
\begin{aligned}
(\tilde{K} \boldsymbol{F})(\boldsymbol{r})= & {\left[\int_{S} \nabla G\left(\boldsymbol{r}, \boldsymbol{r}^{\prime}\right) \times \boldsymbol{F}\left(\boldsymbol{r}^{\prime}\right) d S^{\prime}\right]_{\tan } } \\
(\tilde{D} \boldsymbol{F})(\boldsymbol{r})= & {\left[p . v \cdot \nabla \int_{S} G\left(\boldsymbol{r}, \boldsymbol{r}^{\prime}\right) \nabla^{\prime} \cdot \boldsymbol{F}\left(\boldsymbol{r}^{\prime}\right) d S^{\prime}\right.} \\
& \left.+k^{2} \int_{S} G\left(\boldsymbol{r}, \boldsymbol{r}^{\prime}\right) \boldsymbol{F}\left(\boldsymbol{r}^{\prime}\right) d S^{\prime}\right]_{\tan }
\end{aligned}
$$

for the closed surface $S$ of a volume $D$ and a continuous field $\boldsymbol{F}(\boldsymbol{r})$ tangential to $S$. We also define the single layer potential $(\tilde{S} \boldsymbol{F})(\boldsymbol{r})$ as

$$
(\tilde{S} \boldsymbol{F})(\boldsymbol{r})=\left[\int_{S} G\left(\boldsymbol{r}, \boldsymbol{r}^{\prime}\right) \boldsymbol{F}\left(\boldsymbol{r}^{\prime}\right) d S^{\prime}\right]_{\tan }
$$

to help to write the equations later in a more condensed form.

An ideally corrugated surface only allows electric and magnetic currents to flow to the direction of the corrugation. Both $\boldsymbol{J}(\boldsymbol{r})$ and $\boldsymbol{M}(\boldsymbol{r})$ on an SHS domain can then be written as $\boldsymbol{J}(\boldsymbol{r})=$ $J(\boldsymbol{r}) \hat{\boldsymbol{u}}(\boldsymbol{r}), \boldsymbol{M}(\boldsymbol{r})=M(\boldsymbol{r}) \hat{\boldsymbol{u}}(\boldsymbol{r})$. It is easily seen that the vector product with $\hat{\boldsymbol{n}}$ rotates them to the $\hat{\boldsymbol{v}}$-direction so the last terms on the right-hand sides of (14) and (15) are cancelled out. In the MoM formulation, the basis functions $\boldsymbol{f}_{n}$ for electric and magnetic surface currents for the SHS domains must thus be chosen in such a way that they only carry currents to the direction of the corrugation. This is relatively easy to achieve by using rectangular rooftop functions as basis functions. Since rooftop functions only allow surface currents to flow to the direction of the rectangular elements, by orienting the rectangular elements on the SHS domains to the direction of the corrugation, the flow of the surface currents is automatically restricted to the wanted direction.

For a PEC surface, the usual boundary condition holds, and (14) and (15) reduce to their usual forms. Since the boundary conditions for the PEC and SHS are very close to each other, it is easy to connect SHS patches to PEC surface patches. The connecting is done by correctly choosing the basis functions for the different regions and so that they match on the junctions of the regions. Rectangular elements can also be combined with triangular elements to form hybrid basis functions. On the triangular element, these functions behave as triangle-based Rao-Wilton-Glisson (RWG) functions, and on the rectangular element as rooftop functions. The use of these hybrid functions makes it easy to correctly model nonrectangular piecewise planar structures, such as a triangular corner reflector, by mainly using rooftop functions in order to reduce the number of unknowns compared to the case when solely RWG functions are used. The edges can be modeled by the hybrid functions which avoid the so called staircase effect. 
Next we form the MoM equations for the combined PEC and SHS domains by expanding the electric and magnetic surface currents in (14) and (15) as

$$
\boldsymbol{J}(\boldsymbol{r})=\sum_{n=1}^{N} x_{n} \boldsymbol{f}_{n}(\boldsymbol{r}), \quad \boldsymbol{M}(\boldsymbol{r})=\sum_{n=1}^{N} y_{n} \boldsymbol{f}_{n}(\boldsymbol{r})
$$

and by using the same functions $\boldsymbol{f}_{n}$ as testing functions $\boldsymbol{f}_{m}$, we obtain

$$
\begin{aligned}
& \int_{\operatorname{spt}\left(\boldsymbol{f}_{m}\right)} \boldsymbol{f}_{m}(\boldsymbol{r}) \cdot \boldsymbol{E}^{\mathrm{inc}}(\boldsymbol{r}) d S=-\frac{1}{i \omega \epsilon_{1}} \sum_{n=1}^{N} x_{n} \\
& \quad \times \int_{\mathrm{spt}\left(\boldsymbol{f}_{m}\right)} \nabla \cdot \boldsymbol{f}_{m}(\boldsymbol{r})\left(\tilde{S} \nabla^{\prime} \cdot \boldsymbol{f}_{n}\right)(\boldsymbol{r}) d S \\
& \quad+\frac{k^{2}}{i \omega \epsilon_{1}} \sum_{n=1}^{N} x_{n} \int_{\mathrm{spt}\left(\boldsymbol{f}_{m}\right)} \boldsymbol{f}_{m}(\boldsymbol{r}) \cdot\left(\tilde{S} \boldsymbol{f}_{n}\right)(\boldsymbol{r}) d S \\
& \quad+\sum_{n=1}^{N} y_{n} \int_{\mathrm{spt}\left(\boldsymbol{f}_{m}\right)} \boldsymbol{f}_{m}(\boldsymbol{r}) \cdot\left(\tilde{K} \boldsymbol{f}_{n}\right)(\boldsymbol{r}) d S \\
& \int_{\mathrm{spt}\left(\boldsymbol{f}_{m}\right)} \boldsymbol{f}_{m}(\boldsymbol{r}) \cdot \boldsymbol{H}^{\mathrm{inc}}(\boldsymbol{r}) d S=-\frac{1}{i \omega \mu_{1}} \sum_{n=1}^{N} y_{n} \\
& \quad \times \int_{\mathrm{spt}\left(\boldsymbol{f}_{m}\right)} \nabla \cdot \boldsymbol{f}_{m}(\boldsymbol{r})\left(\tilde{S} \nabla^{\prime} \cdot \boldsymbol{f}_{n}\right)(\boldsymbol{r}) d S \\
& \quad+\frac{k^{2}}{i \omega \mu_{1}} \sum_{n=1}^{N} y_{n} \int_{\mathrm{spt}\left(\boldsymbol{f}_{m}\right)} \boldsymbol{f}_{m}(\boldsymbol{r}) \cdot\left(\tilde{S} \boldsymbol{f}_{n}\right)(\boldsymbol{r}) d S \\
& \quad-\sum_{n=1}^{N} x_{n} \int_{\mathrm{spt}\left(\boldsymbol{f}_{m}\right)} \boldsymbol{f}_{m}(\boldsymbol{r}) \cdot\left(\tilde{K} \boldsymbol{f}_{n}\right)(\boldsymbol{r}) d S .
\end{aligned}
$$

Since we want to analyze the scattering from an electrically very large trihedral corner reflector, the computations are done with the following approximation that further lowers the number of the needed unknowns. We assume that the corner reflector is made of thin triangular plates. For PEC plates, the surface current is then the vector sum of the two sides. For SHS plates, the situation is more complicated, since a real corrugated plate, as illustrated in Fig. 2, can be approximated by an SHS only on the corrugated side since the backside of the plate is made of PEC. Thus, the thin plate impedance boundary model only gives correct results on the corrugated side of the realizable structure. We only model the surface currents on the interior faces of the SHS plates and neglect the induced surface currents on the backside of the SHS plates. The inclusion of the backsides of the SHS plates into the analysis would require us to fix the depth of the corrugation, which is yet a function of the permittivity inside the grooves. The physical realization of the corrugation is considered here to be beyond the scope of this paper. Because the backside of the reflector is largely in shadow from the incident fields and our model is quite large in terms of wavelengths and we only consider the backscattering directions, this customary physical optics type approximation applies and only gives rise to a negligible error in the scattered far fields for the face directions. For sideward or grazing incidence, the discontinuity of the induced currents on the edges may give rise to more substantial errors in the scattered far fields due to the edge diffractions.

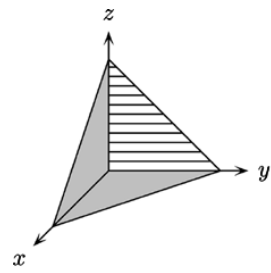

TCR1

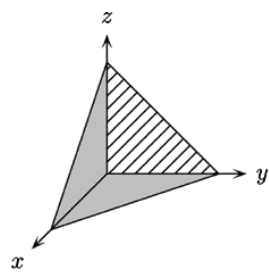

TCR2

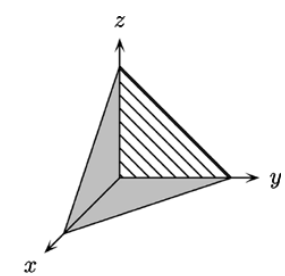

TCR3
Fig. 4. TCR1, TCR2, and TCR3, trihedral corner reflectors with interior $y z$-faces corrugated with conductance vectors $\hat{\boldsymbol{u}}_{1}=\hat{\boldsymbol{y}}, \hat{\boldsymbol{u}}_{2}=(\hat{\boldsymbol{y}}+\hat{\boldsymbol{z}}) / \sqrt{2}$ and $\hat{\boldsymbol{u}}_{3}=(\hat{\boldsymbol{y}}-\hat{z}) / \sqrt{2}$, respectively.

Thus, for sideward directions, the results shown here may not be as accurate as for the face directions.

\section{ANALysis of the BacksCattering PROPERTIES OF the CORNER REFLECTORS BY NUMERICAL RESULTS}

The models for the trihedral corner reflectors were constructed using three isosceles right triangles with cathetus length $a=6 \lambda$ and with frequency $f=1 \mathrm{GHz}$. The models used consisted of more than 3000 elements, including mainly rectangular elements and some triangular elements on the edges of the triangles. The number of basis functions varied between 5000 and 6000 , and they consisted of the rooftop functions on the PEC and SHS domains, of the RWG functions on the inner edges and of the hybrid functions on the outer and inner edges. The exact configuration of these functions depends on the chosen corrugation. Closed-form formulas and accurate numerical integration were used to evaluate the singular integrals in the MoM equations [10], [11].

Five different corrugated corner reflectors were studied: three with one interior corrugated face (reflectors TCR1, TCR2, and TCR3; see Fig. 4), with three different directions for the corrugation, one reflector with two interior corrugated faces (TCR4; see Fig. 10), and one with all the interior faces corrugated (TCR5; see Fig. 10). Different monostatic (i.e., backscattering) and bistatic responses were computed, but to save space only the monostatic conical patterns at $\theta=54.74^{\circ}$ are shown. The incident field was right-handedly circularly polarized in all the examples, except where otherwise noted, with the incident polar angle $\theta=54.74^{\circ}$ and the azimuth angle $\phi$ varying from $0^{\circ}$ to $90^{\circ}$.

The results obtained were verified using an analytical PO method. The trihedral corner reflector was assumed to be made of thin PEC and SHS plates, with the SHS boundary conditions (1) on the SHS plates. The assumptions that were made for the MoM regarding the validity of the $\mathrm{SH}$ surface as a substitute for the ideally tuned corrugated surface also hold for the PO method with the same limitations. The surface currents caused by the wave reflecting multiple times in the reflector were determined using analytical geometry and the reflection dyadic of the SH surface presented in [6]. The PO results suffer from the lack of edge diffractions, especially for grazing incidence, but they are still in good agreement with the results obtained by the MoM computations.

The field strengths shown are normalized with respect to the PEC trihedral corner reflector with the zero level indicating the maximum field strength of the PEC reflector. The field strengths 


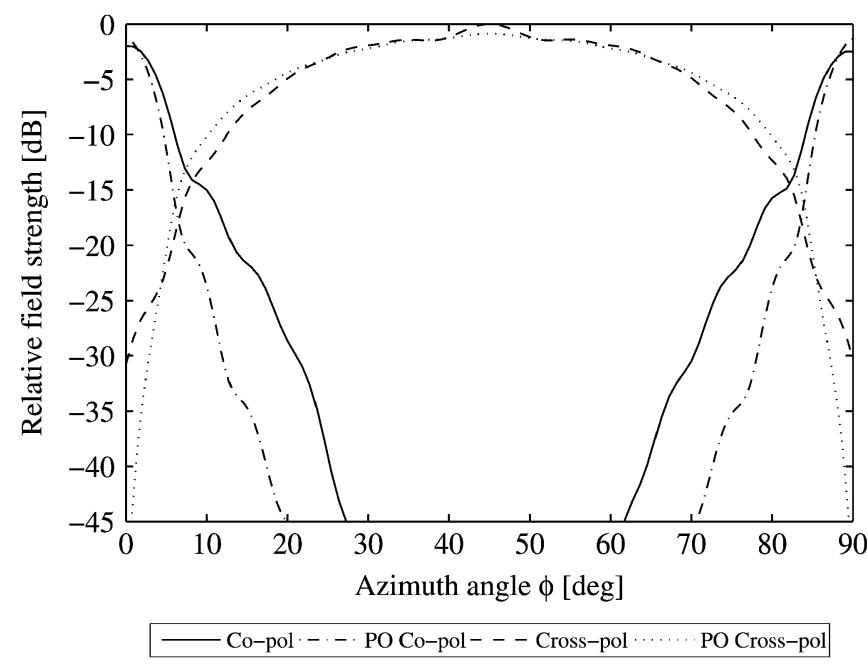

Fig. 5. Relative backscattering co- and cross-polarization responses of the $\mathrm{PEC}$ reflector.

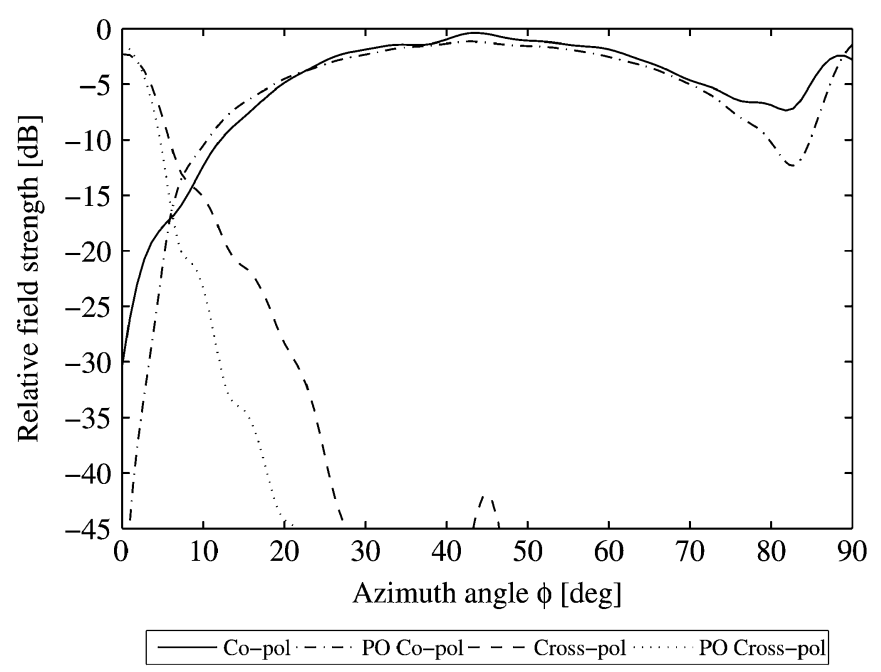

Fig. 6. Relative backscattering co- and cross-polarization responses of the reflector TCR1.

are displayed in decibels with co- and cross-polarization responses shown separately. The PO results are shown alongside the MoM results. The co- and cross-polarization responses are easily computed using the polarization match vector [12].

\section{A. Trihedral Corner Reflector With Corrugated Interior Yz-Face}

Shown in Figs. 5 and 6 are the co- and cross-polarization responses of a PEC trihedral corner reflector and the reflector TCR1. Since in the reflector TCR1 the interior $y z$-face is corrugated along the $y$ axis, its copolarization response is very strong, whereas for the PEC reflector it is very weak in the middle range of the conical backscattering pattern. When only two of the interior faces are illuminated, the PEC reflector shows a good copolarization response. The reflector TCR 1 shows a poor copolarization response when the corrugated face is illuminated along with only one PEC face and a good copolarization response when two PEC faces are illuminated, which agrees well with theoretical predictions in this case.

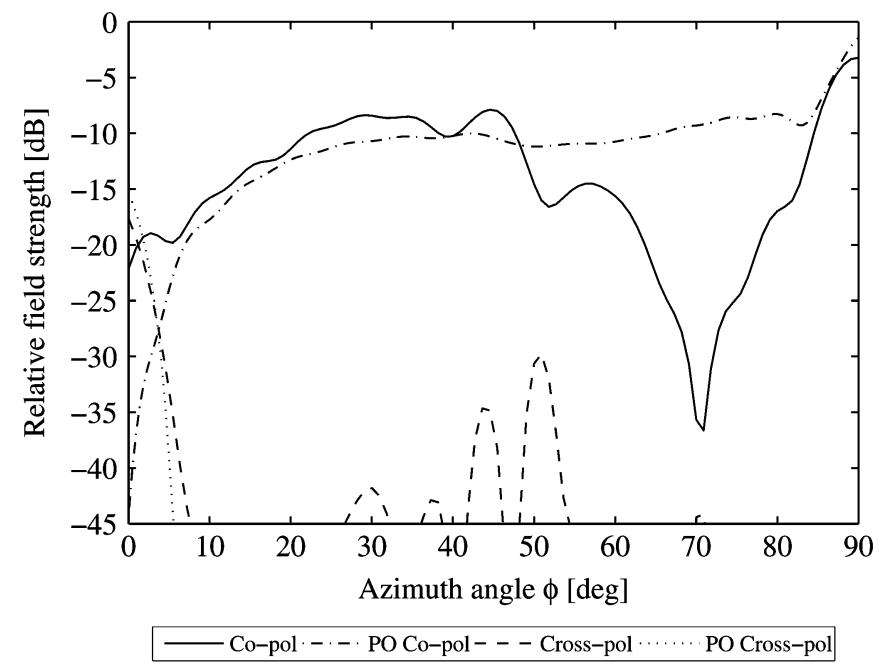

Fig. 7. Relative backscattering co- and cross-polarization responses of the reflector TCR2.

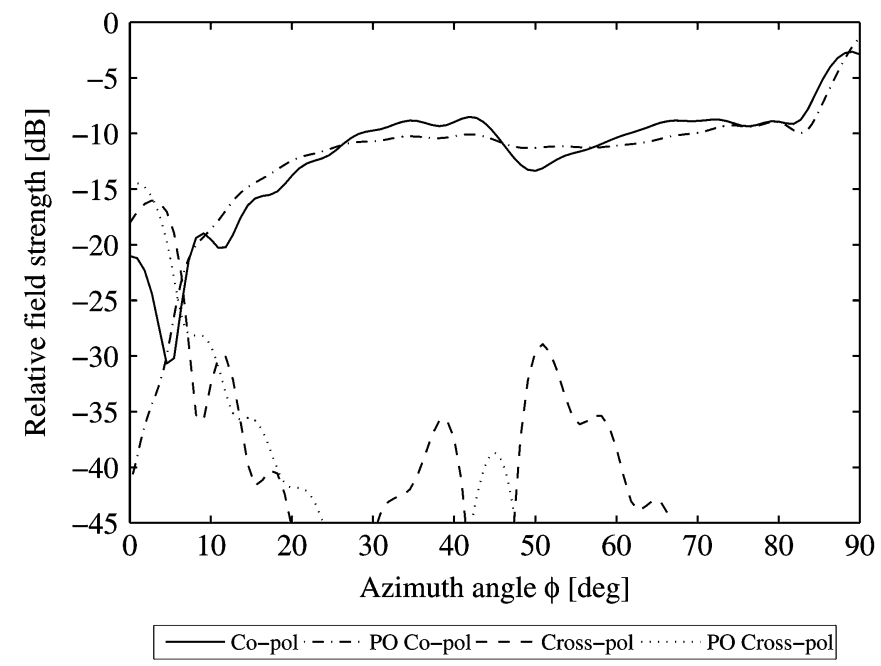

Fig. 8. Relative backscattering co- and cross-polarization responses of the reflector TCR3.

Surprisingly, the intuitive model based on ray theory does not predict, however, that the direction of the corrugation highly affects the backscattering response. The levels of the copolarized backscattered field are much lower for reflectors TCR2 and TCR3 (Figs. 7 and 8), for which the corrugation is rotated $45^{\circ}$ toward or away from the $z$ axis. This has important practical implications, since previously it has been assumed that the direction of the corrugation has little impact on the backscattering response. An explanation for this behavior is not intuitively clear. The most plausible explanation is that the phase of the reflected field from the corrugated interior face changes according to the direction of the corrugation and in some cases the shift in phase results in a deteriorated co-polarization response. Also of interest is the difference between the co-polarization responses of the MoM and the PO method for TCR2, near the azimuth angle $\phi=70^{\circ}$.

Trihedral corner reflectors also have interesting properties regarding linearly polarized incident waves. In Fig. 9 is presented the backscattering response of TCR1 for a vertically linearly polarized incident electric field (i.e., the electric field vector was 


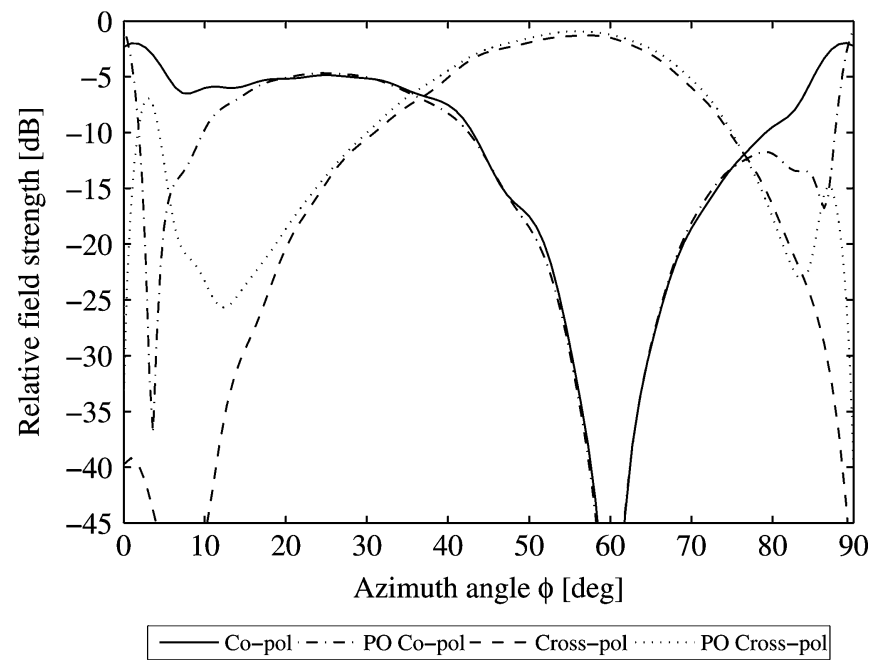

Fig. 9. Relative backscattering co- and cross-polarization responses of the reflector TCR1 for a vertically linearly polarized incident electric field.

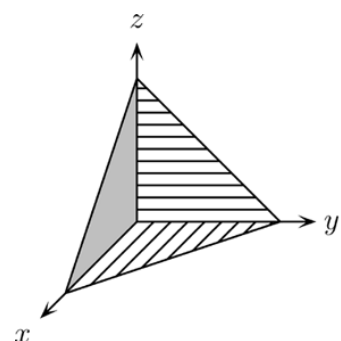

TCR4

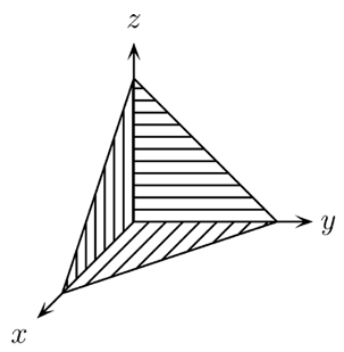

TCR5
Fig. 10. TCR4 and TCR5, trihedral corner reflectors with two interior corrugated faces and all interior faces corrugated, respectively. The conductance vectors are to the directions of the principal axes, i.e., on the $x y$-plane $\hat{\boldsymbol{u}}=\hat{\boldsymbol{x}}$, on the $y z$-plane $\hat{\boldsymbol{u}}=\hat{\boldsymbol{y}}$, and on the $z x$-plane $\hat{\boldsymbol{u}}=\hat{z}$.

in the plane formed by the $z$ axis and the incident wave vector $\boldsymbol{k})$. We see that near the azimuth angle $\phi=60^{\circ}$ direction the cross-polarization response of the reflector is very strong. Also, in the boresight direction, the copolarization response is about $5 \mathrm{~dB}$ lower than for the circularly polarized incident wave.

\section{B. Corner Reflector With Two or Three Corrugated Interior Faces}

We also studied trihedral corner reflectors with two or three corrugated interior faces (reflectors TCR4 and TCR5, respectively; see Fig. 10). According to the theoretical predictions, the reflector TCR4 should exhibit a low copolarization response for circularly polarized incident fields, since the reflections from the corrugated faces retain the handedness of the field but the reflection from the PEC face reverses the handedness of the polarization. Similarly, the reflected field from TCR5 should retain the handedness of the incident field, since the reflections should not modify the polarization.

When two of the interior faces of the corner reflector are corrugated (reflector TCR4), the results again behave in the predicted way (Fig. 11): in the middle range of the backscattering pattern we get three reflections due to all the interior faces' being illuminated, and the copolarization response is remarkably lower than the cross-polarization response. When only the corrugated faces are illuminated, the copolarization response is

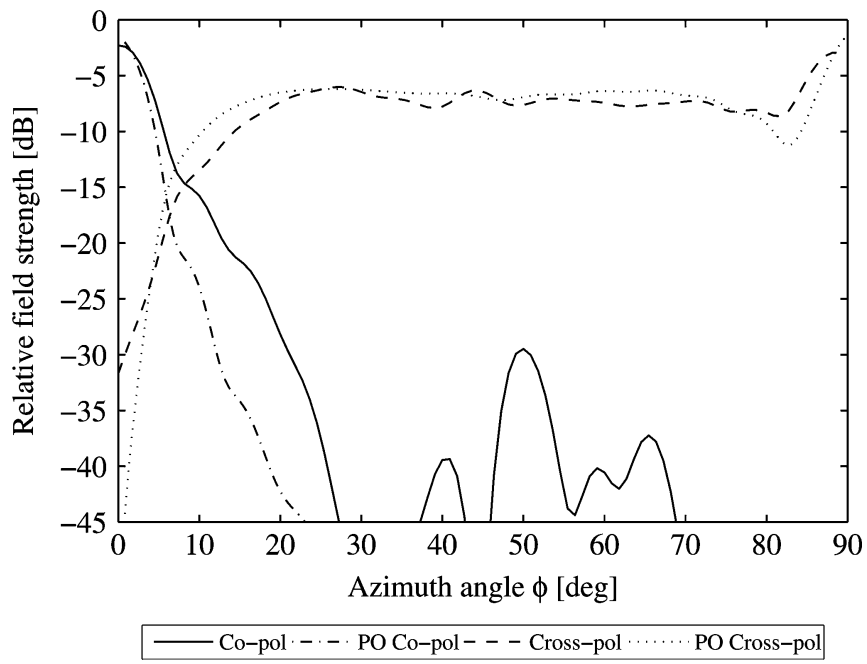

Fig. 11. Relative backscattering co- and cross-polarization responses of the reflector TCR4.

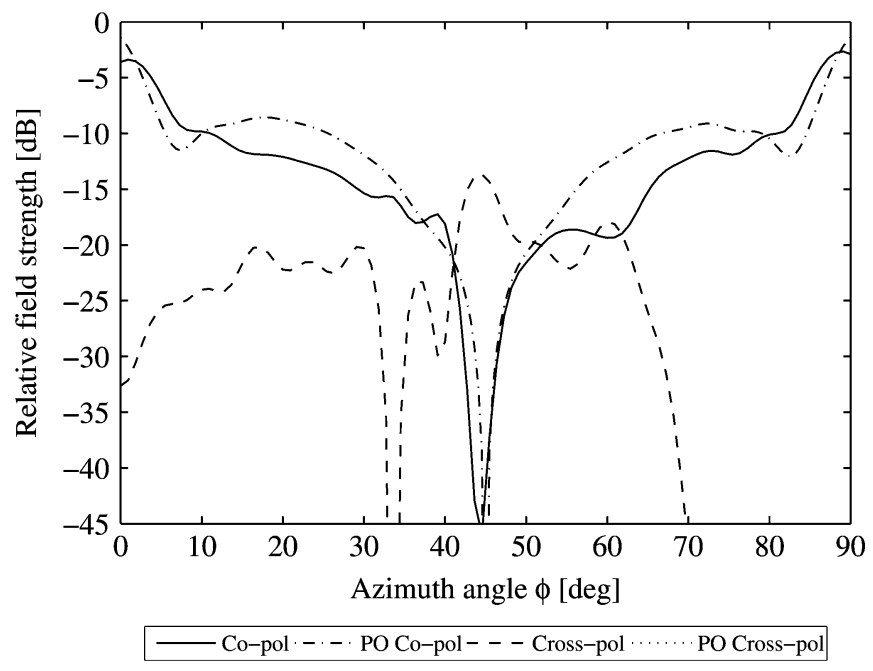

Fig. 12. Relative backscattering co- and cross-polarization responses of the reflector TCR5.

again very high, and similarly when the PEC face and one of the corrugated faces are illuminated, the cross-polarization response is high.

For reflector TCR5, the copolarization response is remarkably lower in the middle range. What is even more significant is that in the boresight direction $\left(\theta=54.74^{\circ}, \phi=45^{\circ}\right)$, the copolarization response vanishes almost entirely (Fig. 12). In the boresight direction, the reflector TCR5 exhibits nearly a PEC reflector-like behavior, i.e., the cross-polarization response is high and the copolarization response is very low. The cross-polarization response of the PO model is zero, which is what the ray theory predicts. The behavior of the copolarization response is again intuitively difficult to explain. It is again more than likely to result from the phase differences of the reflected fields from the three corrugated interior faces.

When the directions of the corrugations of the interior faces are such that from the boresight direction they are at a $120^{\circ}$ angle with respect to each other, as with the reflector TCR5, the phases of the reflected waves from each of the interior faces are such that when they are added together, they destructively 
interfere with each other, resulting in a very poor copolarization response.

\section{CONCLUSION}

A method of moments formulation for soft and hard surfaces has been developed to analyze the backscattering properties of a corrugated trihedral corner reflector. The used formulation treats the corrugated surface as ideally tuned to the incident field frequency.

The focus of this paper has been the polarization response of different corrugation configurations of the corner reflector and how it is affected by the changes in corrugation, particularly for the circularly polarized incident waves. The behavior of the corrugated trihedral corner reflector has some very interesting and sometimes surprising aspects, which are important regarding practical implementations. The obtained results are useful in the wide-ranging use of trihedral corner reflectors in the radar technology or in the remote sensing.

\section{REFERENCES}

[1] P.-S. Kildal, "Definition of artificially soft and hard surfaces for electromagnetic waves," Electron. Lett., vol. 24, no. 3, pp. 168-170, Feb. 1988.

[2] — "Artificially soft and hard surfaces in electromagnetics," IEEE Trans. Antennas Propag., vol. 38, pp. 1537-1544, Oct. 1990.

[3] I. V. Lindell, "Generalized soft-and-hard surface," IEEE Trans. Antennas Propag., vol. 50, pp. 926-929, Jul. 2002.

[4] A. Macikunas and S. Haykin, "Trihedral twist-grid polarimetric reflector," Proc. Inst. Elect. Eng. F, vol. 140, no. 4, pp. 216-222, Aug. 1993.

[5] D. R. Sheen, E. L. Johansen, and L. P. Elenbogen, "The gridded trihedral: A new polarimetric SAR calibration reflector," IEEE Trans. Geosci. Remote Sens., vol. 30, no. 6, pp. 1149-1153, Nov. 1992.

[6] I. V. Lindell and P. P. Puska, "Reflection dyadic for the soft and hard surface with application to the depolarising corner reflector," Proc. Inst. Elect. Eng. Micro. Ant. Propag., vol. 143, no. 5, pp. 417-421, Oct. 1996.

[7] D. G. Michelson and E. V. Jull, "Depolarizing trihedral corner reflectors for radar navigation and remote sensing," IEEE Trans. Antennas Propag., vol. 43, pp. 513-518, May 1995.

[8] C. Gennarelli, G. Pelosi, and G. Riccio, "Physical optics analysis of the field backscattered by a depolarising trihedral comer reflector," Proc. Inst. Elect. Eng. Micro. Ant. Propag., vol. 145, no. 3, pp. 213-218, Jun. 1998.

[9] Z. Ying, P.-S. Kildal, and A. A. Kishk, "Study of different realizations and calculation models for soft surfaces by using a vertical monopole on a soft disk as a test bed," IEEE Trans. Antennas Propag., vol. 44, pp. 1474-1481, Nov. 1996.
[10] R. D. Graglia, "On the numerical integration of the linear shape functions times the 3-D Green's function or its gradient on a plane triangle," IEEE Trans. Antennas Propag., vol. 41, pp. 1448-1455, Oct. 1993.

[11] P. Ylä-Oijala and M. Taskinen, "Calculation of CFIF impedance matrix elements with RWG and $\mathbf{n} \times$ RWG functions," IEEE Trans. Antennas Propag., vol. 51, pp. 1837-1846, Aug. 2003.

[12] I. V. Lindell, Methods for Electromagnetic Field Analysis, ser. Engineering Science. Oxford, U.K.: Oxford Univ. Press, 1992.

Ilari Hänninen received the M.Sc.(Tech.) degree in electrical engineering from Helsinki University of Technology (TKK), Espoo, Finland, in 2002 and the Ingénieur ECP degree from Ecole Centrale Paris, France, in 2002. He is currently pursuing the D.Sc.(Tech.) degree in computational electromagnetics at TKK.

Currently, he is with the Electromagnetics Laboratory, Helsinki University of Technology, as a Research Engineer.

Mikko Pitkonen received the M.Sc. degree in electrical engineering from Helsinki University of Technology (TKK), Espoo, Finland, in 2005.

He is currently a Graduate Student with the Electromagnetics Laboratory, TKK.

Keijo I. Nikoskinen (M'85-SM'00) was born in Kajaani, Finland, in 1962 He received the Dipl.Eng., Lic.Tech., and D.Sc.(Tech.) degrees in electrical engineering from Helsinki University of Technology (TKK), Espoo, Finland, in 1986, 1989, and 1991, respectively.

From 1991 to 1994, he was a Junior Scientist with the Academy of Finland. Since 1996, he has been a Professor of Electromagnetics at TKK. His professional interest covers both the theory and applications of electromagnetics.

Jukka Sarvas (M'98) received the M.Sc. and Ph.D. degrees in mathematics from the University of Helsinki, Finland, in 1968 and 1972, respectively.

He was with the University of Helsinki and with Outokumpu Co. during 1982-1984. In 1988-2002, he was Director of the Rolf Nevanlinna Research Institute of Applied Mathematics and Statistics, University of Helsinki. He was a Visiting Researcher during 1974-1975 and 1979-1980 at the University of Michigan and in 1999-2000 and 2004 at the University of Illinois at Urbana-Champaign. Since 2002, he has been a Professor in computational electromagnetics in the Electromagnetics Laboratory, Helsinki University of Technology. His main interests include field computing with integral equations and fast methods. 